\title{
Mixed Convection MHD Flow of Viscoelastic Fluid in a Porous Medium past a Hot Vertical Plate
}

\author{
Sushil Kumar Ghosh ${ }^{1}$, Gopal Chandra Shit ${ }^{2 *}$ \\ ${ }^{1}$ Department of Mathematics, Garbeta College, Paschim Medinipur, India \\ ${ }^{2}$ Department of Mathematics, Jadavpur University, Kolkata, India \\ Email: 'gopal_iitkgp@yahoo.co.in
}

Received July 18, 2012; revised August 16, 2012; accepted September 2, 2012

\begin{abstract}
The boundary layer flow of a steady incompressible and visco-elastic fluid with short memory (obeying Walters' B fluid model) passing over a hot vertical porous plate has been investigated in the presence of transverse magnetic field. The momentum and energy equations are reduced to couple non-linear partial differential equations along with the boundary conditions by using a suitable similarity transformation. These partial differential equations are transformed to a system of coupled non-linear ordinary differential equations by employing a perturbation technique. The system is solved by developing a suitable numerical procedure such as implicit finite difference scheme along with Newton's linearization method. The computational results for the flow quantities have presented graphically for the effects of thermal radiation, viscous dissipation, heat generation/absorption, visco-elasticity, Hartmann number and the permeability parameter. Results demonstrated that Prandtl number has more pronouncing effect on the temperature distribution rather than the viscosity parameter as well as the thermal radiation parameter. Further the velocity gradient changes significantly due to the presence of temperature dependent variable viscosity.
\end{abstract}

Keywords: MHD Flow; Variable Viscosity; Visco-Elastic Fluid; Viscous Dissipation; Thermal Radiation

\section{Introduction}

The mixed convection boundary layer flow of non-Newtonian fluid in the presence of strong magnetic field has wide range of application in nuclear engineering and industries. In astrophysical and geophysical studies, the MHD boundary layer flows of an electrically conducting fluid through porous media have also enormous applications. These studies are also used for modeling and simulation. Many researchers have studied the transient laminar natural convection flow past a vertical porous plate for the application in the branch of science and technology such as in the field of agriculture engineering and chemical engineering. In petroleum refineries, movement of oil, water and gas through porous media for purification and filtration are bright applied areas of research. With the advancement of science and technology, MHD study on any fluid flow phenomenon exhibits some results which have constructive application for the design of devices. MHD heat transfer has great importance in the liquid metal flows, ionized gas flow in a nuclear reactor and electrolytes. Research works on radiation of heat in natural convection flow are very limited, though these have many modern applications viz. missile

${ }^{*}$ Corresponding author. technology used in army, nuclear power plant, parts of aircraft and ceramic tiles. Thus more investigation is required by considering the terms like magnetic field, heat source/sink, thermal radiation and variable viscosity.

Considering the application in underground water resource and seepage of water under dam, Raptis et al. [1] examined the free convection flow through a vertical porous media. In the other context Raptis et al. [2,3] studied the similar problem by introducing mass transfer, constant suction and constant heat flux. Lai and Kulacki [4] shown an interesting problem regarding free convective flow of Newtonian fluid through a vertical porous medium with varying permeability. Taking into account the temperature dependent viscosity and thermal conductivity Rani and Kim [5] studied the flow of Newtonian fluid over a semi-infinite isothermal vertical cylinder. In the recent past several authors have studied the radiation effect on the flow past a parallel plate [6,7]. Rahman et al. [8] presented an interesting result on a natural convection flow past a vertical plate considering the temperature dependent thermal conductivity and heat conduction. Using implicit finite difference scheme Laganathan et al. [9] solved a coupled non-linear momentum and energy equations for the investigation of the effect of thermal conductivity on the free convective flow over a semi- 
infinite vertical plate under the influence of transverse magnetic field. Shit and Haldar [10] investigated the effects of thermal radiation on the magnetohydrodynamic flow and heat transfer over nonlinear shrinking porous sheet. Jang and Leu [11] analyzed vortex instability in the Newtonian fluid flow and estimated the effect of variable viscosity. Unsteady boundary layer flow over a stretching sheet has rigorously studied by Misra et al. [12] in the presence of variable thermal conductivity. Sharma et al. [13] in two subsequent study showed that the variable permeability increase the flow velocity and heat source has revised effect on Nusselt number for air and water. They elaborately discussed the effect of Grashoff number on Newtonian fluid due to the presence of magnetic field. In polymer processing industries, researchers deals with non-Newtonian fluids in which stress strain relations are non-linear. The differential types of fluid flow exhibits a little effect of deformation gradient on the stress. In the constitutive relations stress is just a function of velocity gradient and its higher power and the stress relaxation time is very small. Rivlin-Eriction fluid shows small relaxation time factor in addition to a convective time derivative in its constitutive relation. The boundary layer flow of non-Newtonian Rivlin-Eriction fluid past a wedge was investigated by Massoudi and Ramezan [14]. They presented results for velocity and shear stress at the wall for different suction/injection rates and different wedge angle.

It is well known that most of the fluid property changes in the presence of heat generation/absorption. Therefore, it is highly meaningful to study the fluid flow and heat transfer taking into account the temperature dependent viscosity. Singh and Gorla [15] carried out the boundary layer analysis of free convective flow of conducting Newtonian fluid over an infinite vertical porous plate. In this investigation they studied the effect of viscous dissipation, Joule heating, thermal diffusion and Hall current. To study the effect of viscosity which varies with temperature and viscous dissipation, Pal and Mondal [16] adopted a problem of mixed convective heat transfer over a stretching surface under the influence of non-uniform heat source/sink. In a similar study Shateyi and Motsa [17] used successive linearization method to solve a set of non-linear ordinary differential equation arises in formulation of unsteady heat transfer over a stretching sheet with Hall Effect. Taking into account the variable viscosity and thermal conductivity Mahanti and Gaur [18] put forwarded some interesting results on free convective flow and heat transfer due to heat sink along an isothermal vertical plate. Numerical solutions for non-Newtonian fluid flow differential equation have been presented by Cortell $[19,20]$. He compared the results with Rajagopal et al. [21] and found close similari- ties. Successively the same author presented similarity solutions when the flow takes place over a stretching sheet. Adopting a second order fluid he studied two cases viz., constant surface temperature and prescribed surface temperature along with the examination of effect of viscoelasticity on heat transfer. Very recently, he studied the viscoelastic fluid flow and heat transfer over a stretching sheet [22]. Incorporating viscous dissipation, work due to deformation, heat radiation and heat generation/absorption terms in energy equation he presented several results and compared among those. Takhar et al. [23] investigated the mixed convection flow over a hot vertical plate in the presence of non-Darcian porous media.

In order to study the flow of viscoelastic fluids of longer relaxation time we consider Walters liquid B' model and investigated the flow and heat transfer through a vertical porous plate in the presence of transverse magnetic field. In the present problem heat and fluid flow has been investigated for viscoelastic fluid when the radiation of heat occurs from the flow domain and flow field has some heat source/sink. Introducing some similarity variables in the governing equations the reducing equations are found to be non-linear partial differential equations. Taking into account the boundary layer approximation length of the plate is adopted as the perturbation parameter. A set of non-linear ordinary differential equations are solved by finite difference techniques with the use of boundary conditions prescribed in the problem. Interesting effects of viscoelasticity, radiation parameter, variable viscosity, magnetic field and heat source are to be observed on account of fluid flow and heat transfer.

\section{Mathematical Formulation and Analysis}

Considering the steady two dimensional flow of viscoelastic fluid past a porous hot vertical plate in which the $x$-axis is taken in the vertical direction and $y$-axis perpendicular to it as horizontal. The fluid is assumed to be falling down vertically over a vertical plane. The constitutive equation of Walter's B' liquid can be written as (cf. Mahapatra and Gupta [24])

$$
\begin{gathered}
\tau_{i k}=-p \delta_{i k}+\tau_{i k}^{\prime} \\
\tau^{\prime i k}(x, t)=2 \int_{-\infty}^{t} \Psi\left(t-t^{\prime}\right) \frac{\partial x^{i}}{\partial x^{\prime m}} \frac{\partial x^{k}}{\partial x^{\prime r}} \mathrm{e}^{(1) m r}\left(x^{\prime}, t^{\prime}\right) \mathrm{d} t^{\prime},
\end{gathered}
$$

where

$$
\Psi\left(t-t^{\prime}\right)=\int_{0}^{\infty} \frac{N(\tau)}{\tau} \mathrm{e}^{-\left(t-t^{\prime}\right) / \tau} \mathrm{d} \tau
$$

$N(\tau)$ is denoted as the distribution function of relaxation time, $\tau_{i j}$ the isotropic stress tensor, $p$ the hydrostatic pressure, $\delta_{i j}$ the metric tensor, $x^{\prime i}$ the position at time $t^{\prime}$ at the point $x^{i}, \mathrm{e}_{i k}^{(1)}$ the rate of strain 
tensor. Taking into account the short relaxation time the Equation (2) becomes

$$
\mathrm{e}_{i k}^{(1)} \tau^{\prime i k}=2 \mu \mathrm{e}^{(1) i k}-2 k_{0} \frac{D}{D t} \mathrm{e}^{(1) i k}
$$

with

$$
\mu=\int_{0}^{\infty} N(\tau) \mathrm{d} \tau, \quad k_{0}=\int_{0}^{\infty} \tau N(\tau) \mathrm{d} \tau
$$

and $\frac{D \Gamma^{i k}}{D t}=\frac{\partial \Gamma^{i k}}{\partial t}+v^{m} \frac{\partial \Gamma^{i k}}{\partial x^{m}}-\frac{\partial v^{k}}{\partial x^{m}} \Gamma^{i m}-\frac{\partial v^{i}}{\partial x^{m}} \Gamma^{m k}$

Considering boundary layer approximation in which the pressure term becomes order of boundary layer thickness $\delta(x)$ and the governing equations such as continuity, momentum and energy equations are reducing to

$$
\begin{gathered}
\frac{\partial u}{\partial x}+\frac{\partial v}{\partial y}=0 \\
u \frac{\partial u}{\partial x}+v \frac{\partial u}{\partial y}=\frac{\mu}{\rho} \frac{\partial^{2} u}{\partial y^{2}}+\frac{\mu}{\rho} \frac{\partial u}{\partial y} \frac{\partial \mu}{\partial y}+g \beta\left(T-T_{\infty}\right) \\
-\frac{g u}{K_{1}^{*}}-\frac{\sigma B_{0}^{2}}{\rho} u-\frac{k_{0}}{\rho}\left(\frac{\partial}{\partial x}\left(u \frac{\partial^{2} u}{\partial y^{2}}\right)\right. \\
\left.+\frac{\partial u}{\partial y} \frac{\partial^{2} v}{\partial y^{2}}+v \frac{\partial^{3} u}{\partial y^{3}}\right) \\
u \frac{\partial T}{\partial x}+v \frac{\partial T}{\partial y}= \\
\lambda \frac{\partial^{2} T}{\partial y^{2}}+\frac{\mu}{\rho C_{P}}\left(\frac{\partial \mu}{\partial y}\right)^{2}-\frac{1}{\rho C_{P}} \frac{\partial q_{r}}{\partial y} \\
+\frac{q^{\prime \prime \prime}}{\rho C_{P}}-\frac{k_{0}}{\rho C_{P}} \frac{\partial u}{\partial y} \frac{\partial}{\partial y}\left(u \frac{\partial u}{\partial x}+v \frac{\partial u}{\partial y}\right)
\end{gathered}
$$

The boundary conditions are

$$
\begin{aligned}
& u=0, v=0, T=T_{w} \text { at } y=0 \\
& u \rightarrow U_{0}, v \rightarrow 0, T \rightarrow 0 \text { when } y \rightarrow \infty
\end{aligned}
$$

where $u$ and $v$ are the velocity components along and perpendicular to the plate, $\mu$ is the viscosity of the fluid, $\rho$ the density of the fluid, $\beta$ the volumetric coefficient of thermal expansion, $K$ the permeability of the porous medium (being a constant), $g$ the field of gravity, $k_{0}$ the visco-elastic constant, $\lambda$ the thermal diffusivity, $C_{p}$ the specific heat at constant pressure, $T$ the temperature variable, $T_{w}$ the constant temperature at the plate, $T_{\infty}$ the ambient temperature, $q_{r}$ is the radiative heat flux and $q^{\prime \prime \prime}$ is the rate of internal heat generation $(>0)$. The second and fifth terms on the right hand side of the Equation (8) represent the effects of viscous dissipation and elastic deformation respectively.

The non-linear radiative heat flux may be expressed as

$$
q_{r}=-\frac{4 \sigma}{3 k} \frac{\partial T}{\partial y}\left(T-T_{\infty}\right)
$$

where $\sigma$ and $k$ are the Stefan Boltzmann constant and mean absorption coefficient respectively.

The term $q^{\prime \prime \prime}$ is considered to be

$$
q^{\prime \prime \prime}=\frac{k u}{v}\left[A^{*}\left(T-T_{\infty}\right) f^{\prime}(\eta)+B^{*}\left(T-T_{\infty}\right)\right]
$$

with $k$, the thermal conductivity, $A^{*}$ and $B^{*}$ are the parameters of space-dependent and temperature dependent internal heat generation $\left(A^{*}>0, B^{*}>0\right)$ and absorption $\left(A^{*}<0, B^{*}<0\right)$ respectively.

The temperature dependent fluid viscosity is given by $\mu=\mu^{*} \mathrm{e}^{-A\left(T-T_{\infty}\right)}$, where $\mu^{*}$ is the constant viscosity away from the ambient of temperature field and $A$ is the con- trolling coefficient.

Let us introduce the similarity variable

$$
\eta=\frac{y}{\delta(x)}
$$

and dimensionless variables $f$ and $\theta$ as follows:

$$
\begin{gathered}
\psi=U_{0} \delta(x) f(\xi, \eta) ; \quad \theta=\frac{T-T_{\infty}}{T_{w}-T_{\infty}} \\
\xi(x)=g \beta \Delta T_{0}\left(x-x_{0}\right) / U_{0}^{2}
\end{gathered}
$$

where,

$$
\Delta T_{0}=T_{w}-T_{\infty} \text { and } \delta(x)=\left[2\left(x-x_{0}\right) v / U_{0}^{2}\right]^{1 / 2}
$$

With the use of above variables into the Equation (6) we obtain

$$
u=\frac{\partial \psi}{\partial y}=U_{0} \frac{\partial f}{\partial \eta}
$$

and

$$
v=-\left[U_{0} f \frac{\mathrm{d} \delta}{\mathrm{d} x}+U_{0} \delta \frac{\partial f}{\partial \xi} \frac{\partial \xi}{\partial x}-\eta U_{0} \frac{\partial f}{\partial \eta} \frac{\mathrm{d} \delta}{\mathrm{d} x}\right]
$$

Introducing the above mentioned non-dimensional variables and $u, v$ from (9) into the Equations (6) and (7) it is found that

$$
\begin{aligned}
& f f^{\prime \prime}+f^{\prime \prime \prime}\left(1-A_{1} \theta\right)-A_{1} f^{\prime \prime} \theta^{\prime}+ \\
& {\left[2 f^{\prime} \frac{\partial f}{\partial \xi}-K_{0}^{*} G_{r}\left(f^{\prime \prime 2}-2 f^{\prime \prime} \frac{\partial^{3} f}{\partial \eta^{2} \partial \xi}-f f^{\prime \prime \prime \prime}-2 f^{\prime \prime \prime \prime} \frac{\partial f}{\partial \xi}\right)\right]_{\xi}} \\
& +2 \theta-K_{1}^{*} f^{\prime}\left(1-A_{1} \theta\right)-\frac{M}{G_{r}} f^{\prime}=0 \\
& 2 \xi P_{r} f^{\prime} \frac{\partial \theta}{\partial \xi}+P_{r} f \theta^{\prime}-2 \xi P_{r} \frac{\partial f}{\partial \xi}+P_{r} \eta f^{\prime} \theta^{\prime} \\
& =\theta^{\prime \prime}+P_{r} E_{c} \xi\left(1-A_{1} \theta\right) f^{\prime \prime 2} \\
& +K_{0}^{*} f^{\prime \prime}\left[f f^{\prime \prime}+f f^{\prime \prime}+f^{\prime \prime} \frac{\partial^{2} f}{\partial \eta \partial \xi}+f^{\prime \prime \prime} \frac{\partial f}{\partial \xi}\right] \\
& +S_{1}\left(\theta^{\prime 2}+\theta \theta^{\prime \prime}\right)-\xi\left(A^{*} f^{\prime}+B^{*} \theta\right)
\end{aligned}
$$


where

$$
\begin{gathered}
P_{r}=\frac{\mu C_{p}}{k} \quad \text { (Prandtl number); } \\
E_{c}=\frac{U_{0}^{2}}{C_{p} \Delta T_{0}} \quad \text { (Eckert number); } \\
G_{r}=\frac{g \beta \Delta T_{0} \nu}{U_{0}} \quad \text { (Grashof number); } \\
M=\frac{\sigma B_{0}^{2} U_{0}}{\rho} \quad \text { (Hartmann number); } \\
K_{0}=k_{0} g \beta U_{0} / k \quad \text { (Viscoelastic parameter); } \\
\frac{4 \sigma \Delta T_{0}}{3 k^{2}}(\text { Thermal radiation parameter); } \\
K_{1}^{*}=\frac{K}{1-\gamma\left(T-T_{\infty}\right)} \\
\varepsilon=\gamma\left(T-T_{\infty}\right), \quad A_{1}=A\left(T_{w}-T_{\infty}\right)
\end{gathered}
$$

Since $\xi$ is a small variable in magnitude so that we can expand the unknown functions in powers of $\xi$ and thus

$$
\begin{gathered}
f(\xi, \eta)=f_{0}(\eta)+\xi f_{1}(\eta)+\xi^{2} f_{2}(\eta)+\cdots \\
\theta(\xi, \eta)=\theta_{0}(\eta)+\xi^{2} \theta_{2}(\eta)+\cdots
\end{gathered}
$$

Using (12) and (13) into (10) and equating the like powers of $\xi$ we get,

$$
\begin{gathered}
f_{0} f_{0}^{\prime \prime}+\left(1-A_{1} \theta_{0}\right) f_{0}^{\prime \prime \prime}-A_{1} f_{0}^{\prime \prime} \theta_{0}^{\prime}=0 \\
f_{1}^{\prime \prime} f_{0}+f_{0}^{\prime \prime} f_{1}+f_{1}^{\prime \prime \prime}\left(1-A_{1} \theta_{0}\right)-A_{1} f_{1}^{\prime \prime} \theta_{0}^{\prime}+2 f_{1} f_{0}^{\prime} \\
+K_{0}^{*} G_{r}\left(2 f_{0} f_{1}^{\prime \prime}+2 f_{0}^{i v} f_{1}-f_{0}^{\prime \prime 2}+f_{0} f_{0}^{i v}\right) \\
+2 \theta_{0}-K_{1}^{*}\left(1-\varepsilon \theta_{0}\right) f_{0}^{\prime}-\frac{M}{G_{r}} f_{0}^{\prime}=0 \\
\left(f_{2}^{\prime \prime} f_{0}+f_{0}^{\prime \prime} f_{2}+2 f_{1} f_{1}^{\prime \prime}\right)+f_{2}^{\prime \prime \prime}\left(1-A_{1} \theta_{0}\right)-A_{1} f_{0}^{\prime \prime} \theta_{2} \\
-A_{1}\left(f_{2}^{\prime \prime} \theta_{0}^{\prime}++f_{0}^{\prime \prime} \theta_{2}^{\prime}\right)+2\left(f_{1} f_{1}^{\prime}+2 f_{2} f_{0}^{\prime}\right) \\
-K_{0}^{*} G_{r}\left(2 f_{0}^{\prime \prime} f_{1}^{\prime \prime}+2 f_{0}^{\prime \prime} f_{2}^{\prime \prime}-f_{1}^{\prime \prime 2}\right. \\
\left.-f_{0} f_{2}^{i v}+f_{2} f_{0}^{i v}+2 f_{1} f_{1}^{i v}-2 f_{2}^{i v} f_{1}-4 f_{2} f_{0}^{i v}\right) \\
+2 \theta_{2}-K_{1}^{*}\left\{\varepsilon f_{0}^{\prime} \theta_{2}^{\prime}-\left(1-\varepsilon \theta_{2}\right) f_{1}^{\prime}\right\}-\frac{M}{G_{r}} f_{1}^{\prime}=0
\end{gathered}
$$

Using (12) and (13) into (11) and equating the coefficients of $\xi^{0}$ and $\xi^{2}$ we get

$$
\begin{aligned}
& \theta_{0}^{\prime \prime}+P_{r} f_{0} \theta_{0}^{\prime}-P_{r} \eta f_{0}^{\prime} \theta_{0}^{\prime}+K_{0}^{*}\left[f_{0}^{\prime} f_{0}^{\prime \prime}+f_{0} f_{0}^{\prime \prime 2}\right. \\
& \left.+2 f_{0}^{\prime \prime 2} f_{1}^{\prime}+2 f_{0}^{\prime \prime 2} f_{1}\right]+R\left(\theta_{0}^{\prime 2}+\theta_{0} \theta_{0}^{\prime \prime}\right)=0
\end{aligned}
$$

$$
\begin{aligned}
& \theta_{2}^{\prime \prime}+P_{r}\left(f_{1} \theta_{0}^{\prime}+f_{0} \theta_{2}^{\prime}\right)-2 P_{r} \theta_{2} f_{0}^{\prime}+2 P_{r} f_{1} \\
& -P_{r} \eta\left(f_{0}^{\prime} \theta_{2}^{\prime}+\theta_{0}^{\prime} f_{1}^{\prime}\right)+P_{r} E_{c}\left(1-A_{1} \theta_{0}\right) f_{0}^{\prime \prime 2} \\
& =+K_{0}^{*}\left[f_{1}^{\prime} f_{0}^{\prime \prime 2}+2 f_{0}^{\prime} f_{0}^{\prime \prime} f_{1}^{\prime \prime}+2 f_{0}^{\prime \prime 2} f_{1}+f_{0}^{\prime \prime 2} f_{0} f^{\prime \prime}\right. \\
& \left.\quad+4 f_{1}^{\prime} f_{1}^{\prime \prime} f_{0}+4 f_{2}^{\prime} f_{0}^{\prime \prime}+f_{1} f_{1}^{\prime \prime} f_{0}^{\prime \prime}+f_{1} f_{0}^{\prime \prime \prime} f_{1}^{\prime \prime}+2 f_{2} f_{0}^{\prime \prime} f_{0}^{\prime \prime \prime}\right] \\
& \quad+R\left(\theta_{0}^{\prime} \theta_{2}^{\prime}+\theta_{2} \theta_{0}^{\prime \prime}+\theta_{2}^{\prime \prime} \theta_{0}\right)-\left(A^{*} f_{0}^{\prime}+A^{*} \theta_{0}^{\prime}\right)
\end{aligned}
$$

and the perturbed boundary conditions reduces to

$$
\begin{aligned}
& f_{0}=f_{1}=f_{2}=0 ; f_{0}^{\prime}=f_{1}^{\prime}=0 ; \theta_{0}=1, \theta_{2}=0 \text { at } \eta=0 \\
& f_{1}^{\prime} \rightarrow 1 ; f_{1}^{\prime} \rightarrow 0, f_{2}^{\prime} \rightarrow 0 ; \theta_{0} \rightarrow 0, \theta_{2} \rightarrow 0 \text { as } \eta \rightarrow \infty
\end{aligned}
$$

Equations (14)-(18) are coupled and highly non-linear ordinary differential equations, findinglexact solutions are not possible. So to get into the insight of the problem, above equations with the boundary conditions (19) and (20) are solved numerically with the use of finite difference scheme developed by Misra and Shit [25].

Another important studies of this investigation are two physical quantities such as local skin friction $\left(C_{f}\right)$ and Nusselt number $\left(N u_{x}\right)$ defined by

$$
C_{f}=\frac{2 \tau_{w}}{\rho U_{0}^{2}} \text { and } N u_{x}=\frac{x q_{x}}{k\left(T_{w}-T_{\infty}\right)}
$$

where

$$
\tau_{w}=\mu\left(\frac{\partial u}{\partial y}\right)_{y=0}
$$

is the wall shear stress and

$$
q_{w}=-k\left(\frac{\partial T}{\partial y}\right)_{y=0}
$$

is the surface heat flux. The non-dimensional form of these two quantities may be expressed as

$$
C_{f} R_{e}^{1 / 2}=2 f^{\prime \prime}(0) \text { and } N u_{x} R_{e}^{1 / 2}=-\theta^{\prime}(0)
$$

\section{Results and Discussions}

The system of coupled non-linear ordinary differential Equations (14)-(18) subject to the boundary conditions (19) and (20) are solved numerically by using the finite difference scheme along with the Newton's linearization technique [25-27] to linearize the discretized equations. The essential features of this technique is that it is based on a finite difference scheme, which has better stability, simple, accurate and more efficient. Finite difference technique leads to a system which is tri-diagonal and therefore speedy convergence as well as economical memory space to store the coefficients.

Figure 1 gives the variation of $f$ along with the distance from the plate. The variation of $A_{1}$, the parameter which is associated with the viscosity function of 
the temperature produce a substantial change in a single valued function $f$ of $\eta$. It is observed that the values of $f$ increases sharply in magnitude as the values of $A_{1}$ increases. In our problem the temperature dependent viscosity vary exponentially with temperature multiplied by a negative constant. The presented graphs are in the sense that as $A_{1}$ increases, the viscosity of the fluid decreases and hence resistance in the flow becomes weaker. It enhances the flow velocity in a substantial manner. Besides, velocity increases at a distance away from the point of commencing the horizontal velocity. This characteristic of velocity appears because the effect of temperature diminishes along with the distance from the plate.

To study the velocity of fluid along the plate for the effect of Grashof number is presented in Figure 2. From this figure we observed that the change is insignificant but the distinction can be made easily. Basic structure of the curves is similar and rectilinear. It is interesting to note that the velocity changes sharply with respect to the distance from the plate. Also the Grashof number reduces the friction then it attributed to increase the flow velocity in moderate magnitude.

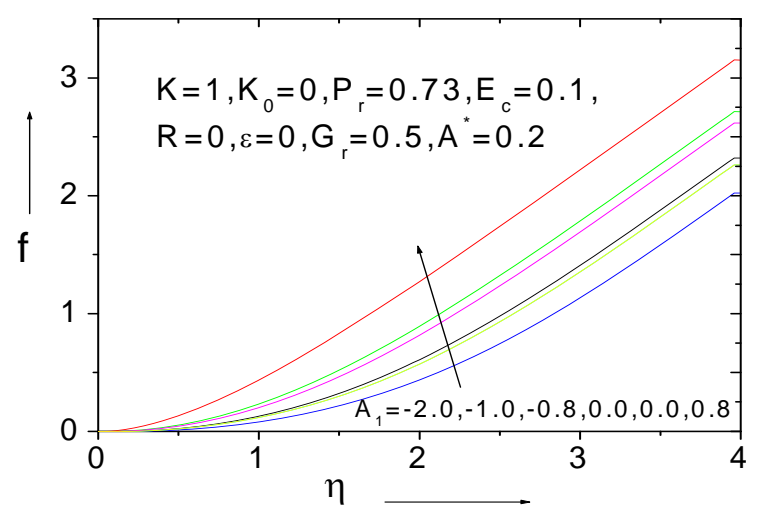

Figure 1. Variation of $f$ with $\eta$ for different values of $A_{1}$.

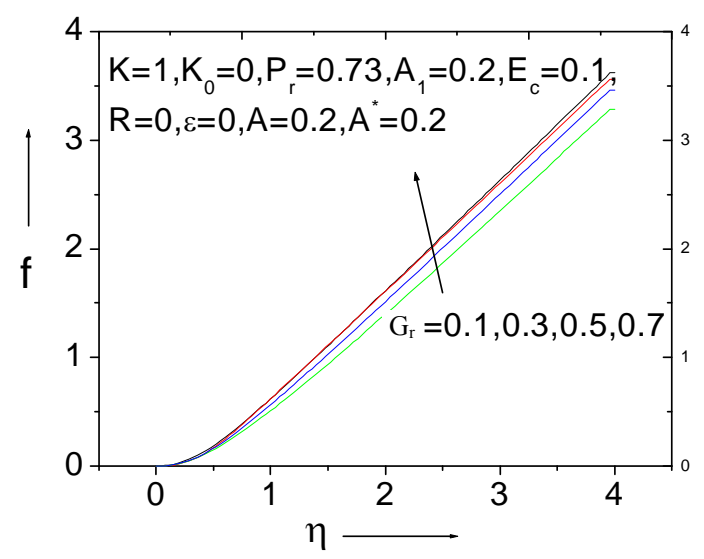

Figure 2. Variation of $f$ with $\eta$ for different values of $G_{r}$.
The temperature profile at different point of the plate along $y$-direction is shown in Figure 3. The effect of increasing the constant $A_{1}$ is prominent on the temperature variation. The temperature at some point on the plate increases with the decreasing values of $A_{1}$. This reveals that the temperature distribution has adverse effect with the viscosity parameter. Thus for any values of $A_{1}$ the temperature distribution vanishes asymptotically. The appreciable change in temperature $(\theta)$ with Prandlt number is found to be presented in Figure 4. It is to be noted that the Prandtl numbers have profound impression in a certain neighborhood of the point of commencement of flow along the horizontal $y$-direction. This is also expected because the Prandtl number is associated with the convective part of the heat transfer equation and the effect of this part on temperature distribution becomes weaker as the fluid moves on the plate for a low Reynolds number flow. Figure 5 represents the temperature variation along the horizontal plate for different radiation parameter. It is observed that the temperature decreases sharply with the increasing distance away from the plate. The sharpness increases with the increasing values of $R$. It is interesting to note from this figure that as heat radiates into the fluid, the temperature increases immediately with a short distance which is quite natural in heat trans-

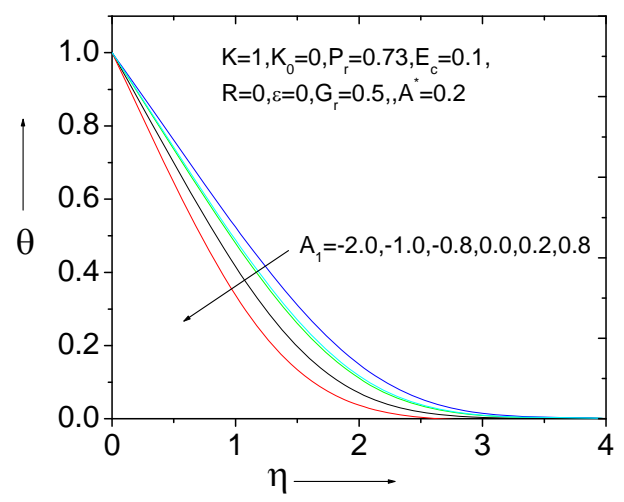

Figure 3. Variation of $\theta$ with $\eta$ for different values of $A_{1}$.

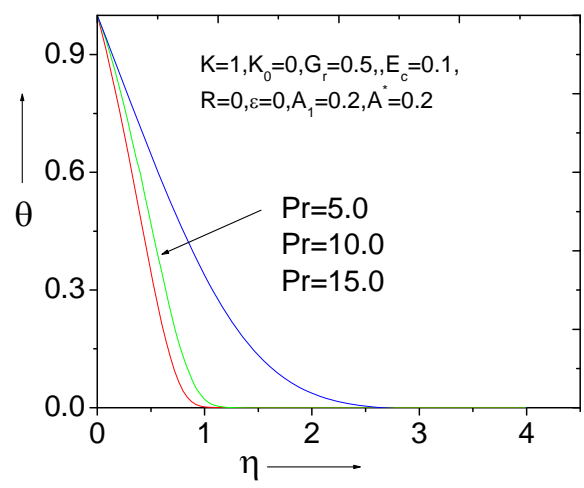

Figure 4. Variation of $\theta$ with $\eta$ for different values of Pr. 
fer can be obtained in Chen [28]. The velocity gradient increases with the increase of the viscosity parameter shown in Figure 6. At some fixed point on the horizontal plate temperature increases with the increasing values of the parameter $A_{1}$. This figure also depicts that the variation of viscosity has significant effect on the velocity gradient. Thus, if viscosity decreases then velocity gradient as well as motion of fluid accelerated. A comparison has also been made with Takhar et al. [23]. Figure 7 represents the variation of $f_{1}^{\prime}$ with the viscosity parameter $A_{1}$. Comparing two Figures 6 and $\mathbf{7}$ it is observe that both the figures have same attitude i.e. $f_{1}^{\prime}$ and $f_{1}^{\prime}$ both decrease with the increasing values of $A_{1}$. For $A_{1}=0$, graphs of Figures $\mathbf{6}$ and $\mathbf{7}$ are totally different. In Figure 7, it may predict that for $A_{1}=-7.0$ or -8.0 , $f_{1}^{\prime}$ becomes zero which is absent in Figure 6. This characteristic appears due to the boundary conditions used to solve for $f_{0}^{\prime}$ and $f_{1}^{\prime}$.

Figure 8 is an interesting figure as this predicts a critical point on the plate after which back flow occur. This figure shows that the velocity gradient $f_{1}^{\prime}$ has positive

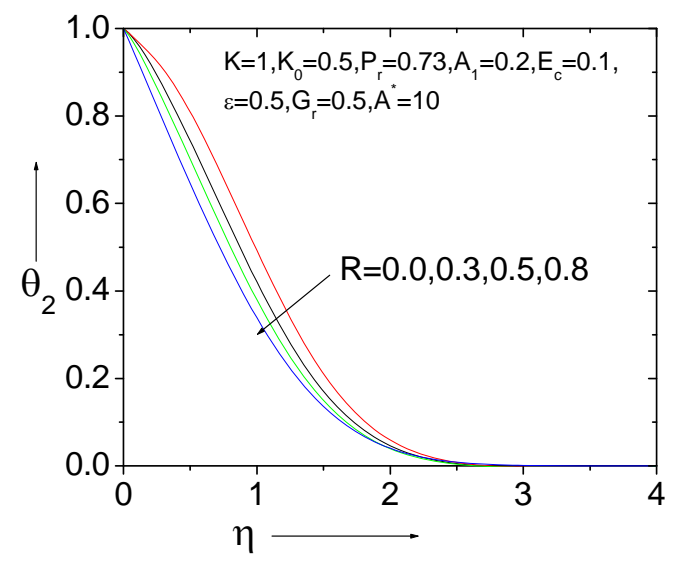

Figure 5. Temperature profile for various values of $\boldsymbol{R}$.

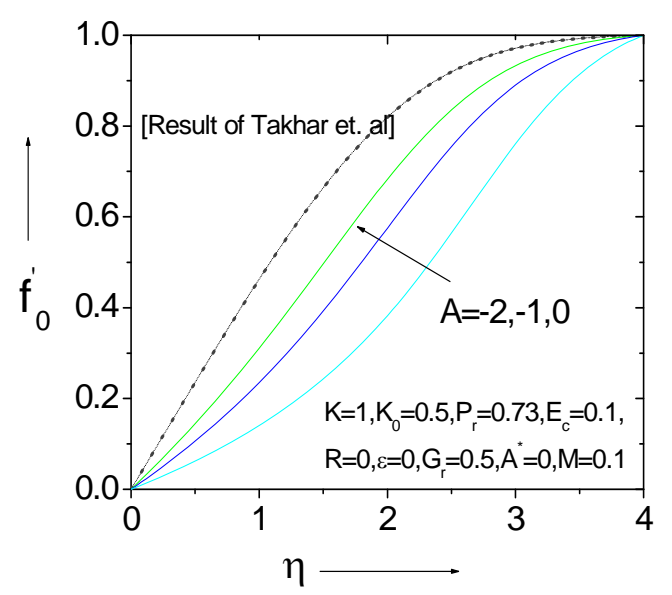

Figure 6. First order velocity distribution for various $A_{1}$.

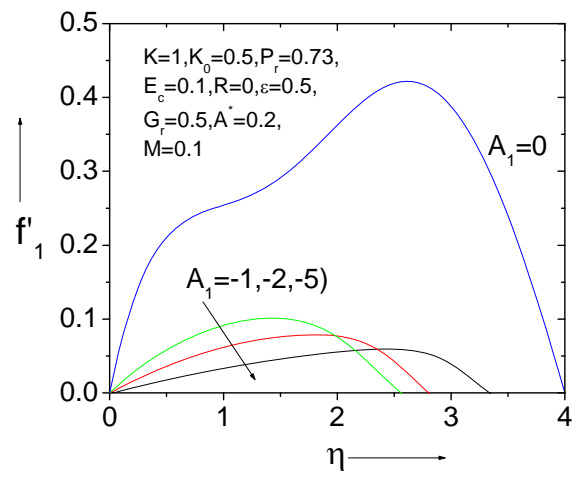

Figure 7. Second order velocity distribution for various $A_{1}$.

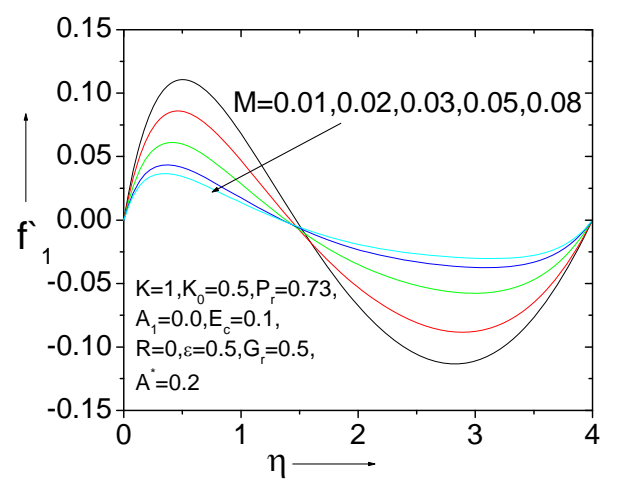

Figure 8. Second order velocity distribution for different values of $M$.

and negative values before and after $\eta=1.5$.

It is worthwhile to mention here that $f_{1}^{\prime}$ decreases with the increasing values of $M$. Thus it may conclude that the influence of magnetic field on the velocity gradient is predominantly vanishes after certain distance from the plate.

Figure 8 is an interesting figure as this predicts a critical point on the plate after which back flow occur. This figure shows that the velocity gradient $f_{1}^{\prime}$ has positive and negative values before and after $\eta=1.5$.

It is worthwhile to mention here that $f_{1}^{\prime}$ decreases with the increasing values of $M$. Thus it may conclude that the influence of magnetic field on the velocity gradient is predominantly vanishes after certain distance from the plate.

In the present study we have considered a flow past a porous vertical plate where the permeability is a function of $\theta$. Figure 9 gives the distribution of second order temperature for various permeability constants. The structure of this figure is similar for positive and negative values of $\varepsilon$ but the trend is reversed in the case of its values in magnitude. It is found that the effect of $\varepsilon$ is more prominent in the vicinity of the vertical wall and it is nearly same for $\eta=1.0$ onwards. Therefore heating and cooling both are possible by controlling the values of 
$\varepsilon$. The Grashof number plays an important role on the flow and heat transfer of a viscoelastic fluid. To interpret the effect of $\mathrm{Gr}$ on second order velocity gradient the Figure 10 is being presented. This observation illustrates that $f_{1}^{\prime}$ decreases slowly with the decreasing values of Gr. Also the values of $f_{1}^{\prime}$ cannot become zero at any point on the horizontal plate as well as for any value of Gr. The second order temperature distribution with $\eta$ for different values of $G_{r}$ has been presented in Figure 11. For the same values of $G_{r}$, there is always cooling and heating in the fluid before and after the plate positioned at $\eta=0.5$.

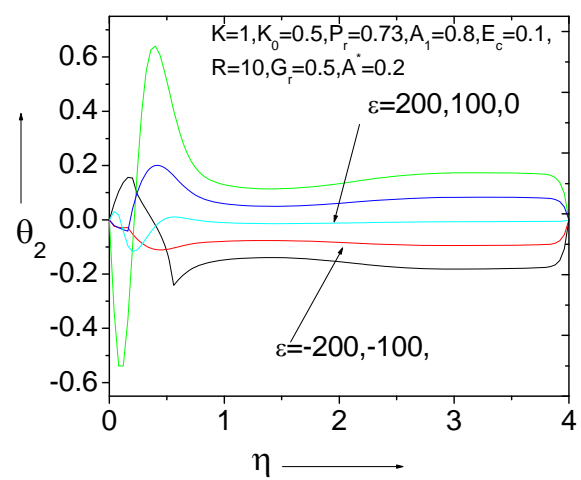

Figure 9. Second order velocity distribution for various $M$.

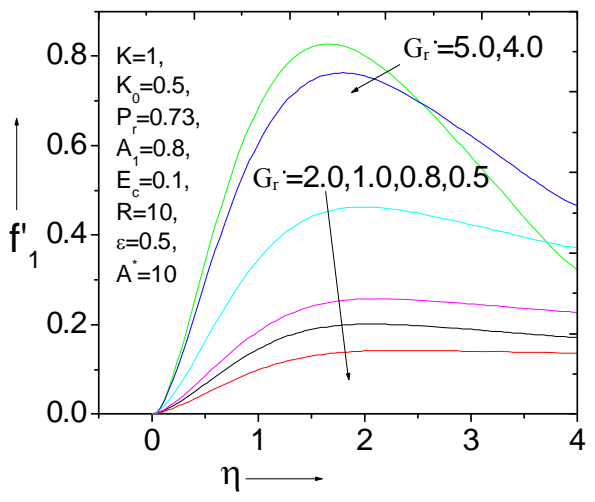

Figure 10. Second order velocity for various $G_{r}$.

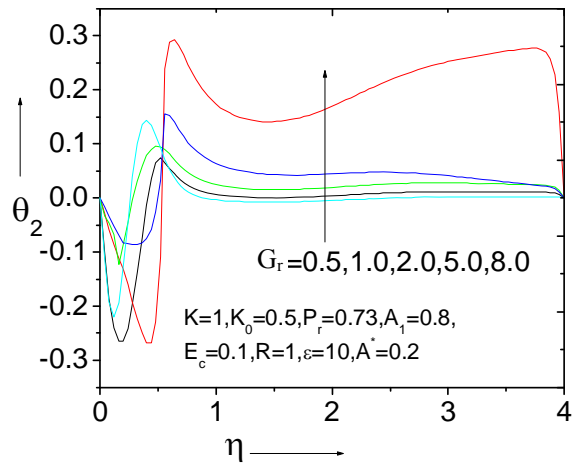

Figure 11. Second order temperature profile for various $G_{r}$.
Figure 12 gives the variation of second order velocity component for different permeability parameter $K$. It is seen that $f_{1}$ decrease with the increasing values of $K$. Here the permeability of the fluid effectively decreases the flow velocity. It is interesting to mention here that the fluid velocity can never be zero as we advance along the plate. Figure 13 depicts the change in $f_{1}$ for various values of the viscoelastic parameter $K_{0}$. With the increasing values of $K_{0}$ back flow becomes more prominent. It is observed that at the beginning of motion flow appears to be positive; however, with the advancement of the flow over the horizontal plate back flow appears. Therefore the viscoelastic parameter retards the motion significantly. To furnish the influence of source and sink parameter $A^{*}$ on $\theta_{2}$, we presented it in Figure 14. This parameter has mild effect on the second order temperature distribution, however in the neighborhood of the vertical plate, the magnitude of $\theta_{2}$ has substantial distinction between different curves. Also, for the critical value $\eta=1.0, \theta_{2}$ becomes negative i.e. there is cooling in the fluid which is an important characteristic. Thus, for positive source constant, $A^{*}$ fluid may lose its heat after crossing over the critical distance.

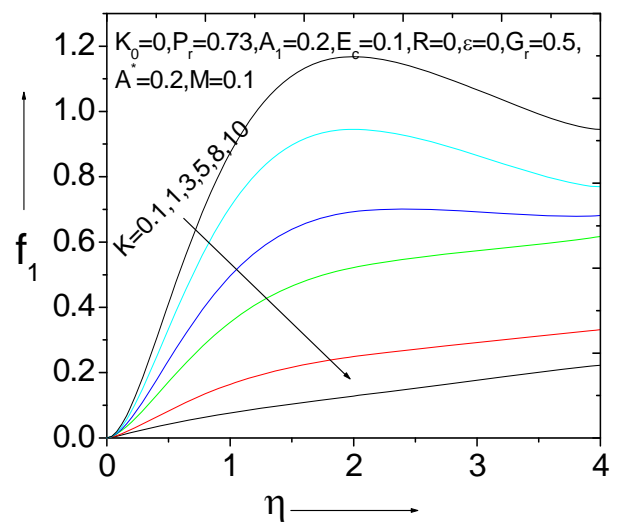

Figure 12. Variation of $f_{1}$ for various values of $K$.

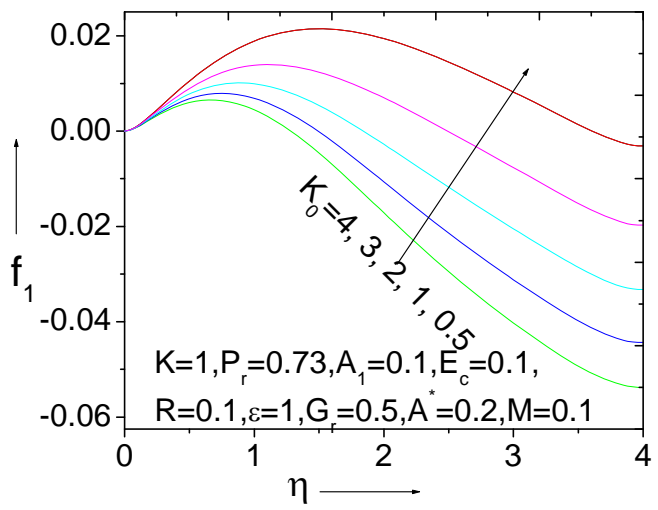

Figure 13. Variation of $f_{1}$ for various $K_{0}$. 


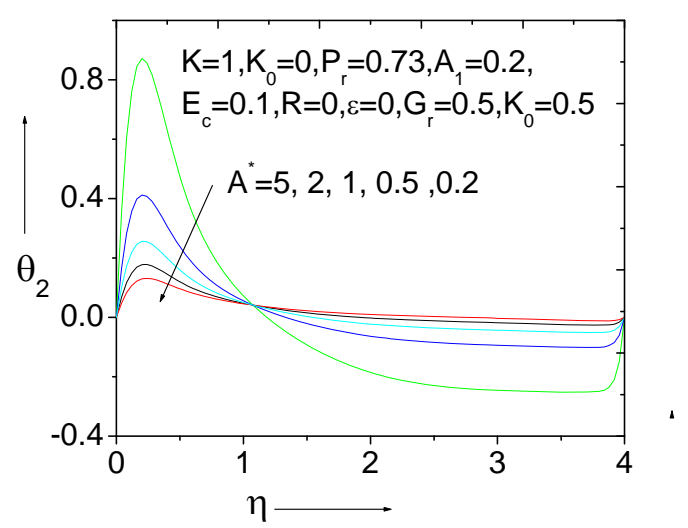

Figure 14. Second order temperature profile for different $A^{*}$.

From Table 1 it is found that the zeroth order and first order skin-friction varies significantly with various viscosity parameter. As the exponential coefficient of viscosity increase when other parameters are fixed, the magnitude of frictional resistance increase on the plate. Also it has decreasing effect for the increasing values of magnetic parameter because this parameter creates more resistance on the flow. Table 2 illustrates the variation of
Nusselt number for different values of the viscosity parameter $A_{1}$. We observed that the rate temperature distribution in the fluid increases with the increasing values of viscosity parameter. This is due to the fact that the viscosity parameter depends on temperature. Moreover, the magnitude of local Nusselt number decreases with the increasing of radiation parameter.

\section{Conclusion}

In the present paper a numerical investigation has been conducted for the flow of visco-elastic fluid past a porous vertical plate in the presence of transverse magnetic field. Results are presented graphically for different non-dimensional parameters such as $\mathrm{M}$ the magnetic parameter (Hartmann number), $G_{r}$ the Grashof number, $E_{c}$ the Eckert number, $A_{1}$ the constant appeared in the exponentially variable viscosity, $R$ the radiation parameter, $A^{*}$ the source parameter, $K_{0}$ the visco-elastic parameter and $K$ the permeability parameter. Using perturbation technique the momentum and energy equations are reduced to a coupled non-linear ordinary differential equation and those are solved by finite difference method by already developed scheme. Results extracted from this

Table 1. Values of skin-friction coefficient $f_{0}^{\prime \prime}(0), f_{1}^{\prime \prime}(0)$ for different values of viscosity parameter $A$ and magnetic parameter $M$ with $K=1.0, K_{0}=0.5, E_{c}=0.1, R=0.0, \varepsilon=0.0, G_{r}=0.5, A^{*}=0.2, M=0.2$.

\begin{tabular}{cccccccccccc}
\hline$A_{1}$ & & -5.0 & & & -3.0 & \multicolumn{3}{c}{0.0} & & & 0.2 \\
\hline$M$ & $f_{0}^{\prime \prime}(0)$ & $f_{1}^{\prime \prime}(0)$ & $f_{0}^{\prime \prime}(0)$ & $f_{1}^{\prime \prime}(0)$ & $f_{0}^{\prime \prime}(0)$ & $f_{1}^{\prime \prime}(0)$ & $f_{0}^{\prime \prime}(0)$ & $f_{1}^{\prime \prime}(0)$ \\
0.1 & 0.8341 & 1.4520 & 1.0034 & 3.2981 & 6.8743 & 9.2897 & 11.4532 & 13.0943 \\
0.5 & 0.7234 & 1.0756 & 1.3420 & 2.4861 & 4.2091 & 7.9824 & 11.0126 & 13.0018 \\
0.7 & 0.6213 & 1.0002 & 1.0217 & 1.8749 & 3.6849 & 6.4320 & 9.9682 & 11.9828 \\
0.9 & 0.5103 & 0.8321 & 0.9092 & 1.0502 & 2.4703 & 5.6027 & 7.2581 & 10.3904 \\
1.2 & 0.4579 & 0.7480 & 0.7769 & 0.8062 & 1.6729 & 4.6802 & 6.0361 & 8.3096 \\
1.5 & 0.2495 & 0.6103 & 0.5302 & 0.5481 & 0.7302 & 2.6819 & 5.0318 & 6.9906 \\
\hline
\end{tabular}

Table 2. Numerical values of the local Nusselt number $\theta_{0}^{\prime}(0), \theta_{2}^{\prime}(0)$ for different viscosity parameter $A_{1}$ and magnetic parameter $M . K=1.0, K_{0}=0.5, E_{c}=0.1, R=0.0, \varepsilon=0.0, G_{r}=0.5, A^{*}=0.2, M=0.2$.

\begin{tabular}{|c|c|c|c|c|c|c|c|c|}
\hline \multirow{2}{*}{$\begin{array}{l}A_{1} \\
R\end{array}$} & \multicolumn{2}{|c|}{-5.0} & \multicolumn{2}{|c|}{-3.0} & \multicolumn{2}{|c|}{0.0} & \multicolumn{2}{|c|}{0.2} \\
\hline & $\theta_{0}^{\prime}(0)$ & $\theta_{2}^{\prime}(0)$ & $\theta_{0}^{\prime}(0)$ & $\theta_{2}^{\prime}(0)$ & $\theta_{0}^{\prime}(0)$ & $\theta_{2}^{\prime}(0)$ & $\theta_{0}^{\prime}(0)$ & $\theta_{2}^{\prime}(0)$ \\
\hline 0.1 & 0.3591 & 2.5020 & 1.7310 & 3.2082 & 4.0835 & 6.0807 & 8.0391 & 10.021 \\
\hline 0.3 & 0.3109 & 2.0031 & 1.1200 & 2.9080 & 3.6117 & 5.8024 & 7.0816 & 9.0826 \\
\hline 0.9 & 0.2713 & 1.5514 & 0.8279 & 1.7999 & 3.0010 & 5.1320 & 6.0321 & 7.9397 \\
\hline 1.5 & 0.1030 & 0.9831 & 0.2036 & 0.8593 & 1.7903 & 4.2731 & 5.5081 & 6.4501 \\
\hline 2.3 & 0.0709 & 0.3804 & 0.1005 & 0.3362 & 0.2419 & 2.0255 & 3.6091 & 4.0906 \\
\hline 2.8 & 0.0125 & 0.1038 & 0.0502 & 0.0998 & 0.07677 & 0.9512 & 1.0085 & 1.9862 \\
\hline
\end{tabular}


study concluded that the temperature distribution as well as fluid velocity substantially depends on variable viscosity parameter which is the function of temperature. Another important feature is the paramount effect of Grashof number on the temperature and velocity. Heat radiation into the fluid enhances the temperature significantly in this study.

\section{REFERENCES}

[1] A. Raptis, C. Perdikis and G. Tzivanidis, "Free Convection Flow through a Porous Medium Bounded by a Vertical Surface," Journal of Physics D: Applied Physics, Vol. 14, No. 7, 1981, pp. 99-102. doi:10.1088/0022-3727/14/7/001

[2] A. Raptis and G. Tzivanidis "Free Convection and Mass Transfer Flow through a Porous Medium Bounded by an Infinite Vertical Limiting Surface with Constant Suction," Letters in Heat and Mass Transfer, Vol. 8, No. 5, 1981, pp. 417-424. doi:10.1016/0094-4548(81)90029-1

[3] A. Raptis, N. Kafousias and C. Massalas, "Free Convection and Mass Transfer Flow through a Porous Medium Bounded by an Infinite Vertical Limiting Surface with Constant Heat Flux," Journal of Applied Mathematics and Mechanics (ZAMM), Vol. 62, 1982, pp. 489-491.

[4] F. C. Lai and F. A. Kulacki, "The Effect of Variable Viscosity on Convective Heat Transfer along a Vertical Surface in a Saturated Porous Media," International Journal of Heat and Mass Transfer, Vol. 33, No. 5, 1990, pp. 1028-1031. doi:10.1016/0017-9310(90)90084-8

[5] H. P. Rani and C. N. Kim, "Transient Convection on Vertical Cylinder with Variable Viscosity and Thermal Conductivity," Journal of Thermo Physics and Heat Transfer, Vol. 22, No. 2, 2008, pp. 254-261. doi:10.2514/1.32501

[6] P. K. Sharma, "Simultaneous Thermal and Mass Diffusion in Three Dimensional Mixed Convection Flow through a Porous Media," Journal of Porous Media, Vol. 8, No. 4, 2005, pp. 409-417. doi:10.1615/JPorMedia.v8.i4.70

[7] A. Raptis and C. Perdikis, "Viscoelastic Flow by the Presence of Rotation," Journal of Applied Mathematics and Mechanics (ZAMM), Vol. 78, No. 4, 1998, pp. 277279.

[8] M. M. Rahman, A. A. Mamun and M. A. Azim, "Effects of Temperature Dependent Thermal Conductivity on MHD Free Convective Flow along a Vertical Flat Plate with Heat Conduction," Non-Linear Analysis Modelling and Controll, Vol. 13, No. 4, 2008, pp. 513-524.

[9] P. Laganathan, P. Ganesan and D. Iranian, "Effects of Thermal Conductivity on Unsteady MHD Free Convective Flow over a Semi-Infinite Vertical Plate," International Journal of Engineering Sciences, Vol. 52, No. 11, 2010, pp. 6257-6268.

[10] G. C. Shit and R. Haldar, "Effects of Thermal Radiation on MHD Viscous Fluid Flow and Heat Transfer over Non-Linear Shrinking Porous Sheet," Applied Mathematics and Mechanics, Vol. 32, No. 6, 2011, pp. 677-688. doi:10.1007/s10483-011-1448-6
[11] J.-Y. Jang and J.-S. Leu, "Variable Viscosity Effects on the Vortex Instability of Free Convection Boundary Layer over a Horizontal Surface," Numerical Heat Transfer, Vol. 25, No. 4, 1994, pp. 495-500. doi:10.1080/10407789408955962

[12] M. Misra, N. Ahmad and Z. U. Siddiqui, "Unsteady Boundary Layer Flow Past a Stretching Plate and Heat Transfer with Variable Thermal Conductivity," World Journal of Mechanics, Vol. 2, No. 1, 2012, pp. 35-41. doi:10.4236/wjm.2012.21005

[13] B. K. Sharma, R. C. Chaudhury and M. Agarwal, "Radiation Effect of Steady Free Convective Flow along a Uniform Moving Porous Vertical Plate in Presence of Heat Source/Sink and Transverse Magnetic Field," Bulletin of Calcutta Mathematical Society, Vol. 100, 2008, pp. 529538.

[14] M. Massoudi and M. Ramezan, "Effect of Injection or Suction on the Falkner-Skan Flows of Second Grade Fluids," International Journal of Non-Linear Mechanics, Vol. 24, No. 3, 1989, pp. 221-227. doi:10.1016/0020-7462(89)90041-3

[15] A. K. Singh and R. S. R. Gorla, "Free Convective Heat and Mass Transfer with Hall Current, Joule Heating and Thermal Diffusion," Heat and Mass Transfer, Vol. 45, No. 11, 2009, pp. 1341-1349. doi:10.1007/s00231-009-0506-9

[16] D. Pal and H. Mondal, "Effect of Variable Viscosity on MHD Non-Darcy Mixed Convective Heat Transfer over a Stretching Sheet Embedded in a Porous Medium with Non-Uniform Heat Source/Sink," Communications in NonLinear Science and Numerical Simulation, Vol. 15, No. 6, 2010, pp. 1553-1564. doi:10.1016/j.cnsns.2009.07.002

[17] S. Shateyi and S. S. Motsa, "Variable Viscosity on Magnetohydrodynamic Fluid Flow and Heat Transfer over an Unsteady Stretching Surface with Hall Effect," Boundary Value Problems, Vol. 1, 2010, pp. 1-20.

[18] N. C. Mahanti and P. Gaur, "The Effect of Varying Viscosity and Thermal Conductivity on Steady Free Convective Flow and Heat Transfer along an Isothermal Vertical Plate in the Presence of Heat Sink," Journal of Applied Fluid Mechanics, Vol. 2, No. 1, 2009, pp. 23-28.

[19] R. Cortell, "Similarity Solutions for Flow and Heat Transfer of a Viscoelastic Fluid over a Stretching Sheet," International Journal of Non-Linear Mechanics, Vol. 29, No. 2, 1994, pp. 155-161.

[20] R. Cortell, "Numerical Solutions for the Flow of a Fluid of Grade Three Past an Infinite Porous Plate," International Journal of Non-Linear Mechanics, Vol. 28, No. 6, 1993, pp. 623-626. doi:10.1016/0020-7462(93)90023-E

[21] K. R. Rajagopal, A. Z. Szeri and W. Troy, "An Existence Theorem for the Flow of a Non-Newtonian Fluid Past an Infinite Porous Plate," International Journal of Non-Linear Mechanics, Vol. 21, No. 4, 1986, pp. 279-289. doi:10.1016/0020-7462(86)90035-1

[22] R. Cortell, "Viscoelastic Fluid Flow and Heat Transfer over a Stretching Sheet under the Effects of a Non-Uniform Heat Source, Viscous Dissipation and Thermal Radiation," International Journal of Heat and Mass Transfer, Vol. 50, No. 15-16, 2007, pp. 3152-3162. 
doi:10.1016/j.ijheatmasstransfer.2007.01.003

[23] H. S. Takhar, V. M. Soundalgekar and A. S. Gupta, "Mixed Convection of an Incompressible Viscous Fluid in a Porous Medium Past a Hot Vertical Plate," International Journal of Non-Linear Mechanics, Vol. 25, No. 6, 1990, pp. 723-728. doi:10.1016/0020-7462(90)90010-7

[24] T. R. Mahapatra and A. S. Gupta, "Stagnation-Point Flow of a Viscoelastic Fluid towards a Stretching Surface," International Journal of Non-Linear Mechanics, Vol. 39, No. 5, 2004, pp. 811-820.

doi:10.1016/S0020-7462(03)00044-1

[25] J. C. Misra and G. C. Shit, "Flow of a Biomagnetic Visco-Elastic Fluid in a Channel with Stretching Walls,"
ASME Journal of Applied Mechanics, Vol. 76, No. 6, 2009, pp. 1-9. doi:10.1115/1.3130448

[26] T. Cebeci and J. Cousteix, "Modeling and Computation of Boundary-Layer Flows," Springer-Verlag, Berlin, 1999

[27] J. C. Misra and G. C. Shit, "Biomagnetic Viscoelastic Fluid Flow over a Stretching Sheet," Applied Mathematics and Computation, Vol. 210, No. 2, 2009, pp. 350-361. doi:10.1016/j.amc.2008.12.088

[28] C.-H. Chen, "Combined Effects of Joule Heating and Viscous Dissipation on MHD Flow Past a Permeable, Stretching Surface with Free Convection and Radiative Heat Transfer," ASME Journal of Heat Transfer, Vol. 132, No. 6, 2010, pp. 1-3. doi:10.1115/1.4000946 